\title{
Thyroid Follicular Epithelial Cell-Derived Cancer: New Approaches and Treatment Strategies
}

\author{
Julie Bolin \\ Nuclear Medicine Technology Program, GateWay Community College, Phoenix, Arizona
}

\begin{abstract}
Thyroid follicular epithelial cell-derived cancer includes papillary carcinoma, follicular carcinoma, Hürthle cell carcinoma, poorly differentiated thyroid cancer, and anaplastic thyroid carcinoma. Although the incidence of thyroid cancer has increased over the past 30 years, there has not been a significant increase in patient mortality. Use of increasingly sensitive detection methods such as high-resolution imaging has enabled earlier detection and better characterization of the thyroid malignancies. In the past several years, researchers have evaluated genetic mutations promoting thyroid carcinogenesis and oncogenesis. The identification of genetic mutations is important in understanding tumor initiation and progression. Additionally, these identified mutations may also serve as potential diagnostic or prognostic indicators and therapeutic molecular targets.
\end{abstract}

Key Words: anaplastic thyroid carcinoma; follicular thyroid carcinoma; papillary thyroid carcinoma; mitogen activated protein kinase; ATC; FTC

J Nucl Med Technol 2021; 49:199-208

DOI: $10.2967 /$ jnmt.120.257105

$\mathbf{T}$ hyroid cancer is the twelfth most common type of cancer in the United States. According to the National Cancer Institute, over 850,000 people were living with thyroid cancer in the United States in 2017, and it was estimated that there would be 52,890 new cases of thyroid cancer in 2020 (1). Women are disproportionately affected across all age groups, with thyroid cancer having a 3 times higher incidence in women than in men. Thyroid cancer is more common in adults over $30 \mathrm{y}$ old, with the peak incidence occurring in women in their 40s-50s and men in their 60s-70s (2). Thyroid cancer rarely affects children and adolescents; however, it accounts for $1.4 \%$ of pediatric malignancies (3). The incidence of thyroid cancer has also increased worldwide but may be variable because of variations in health-care access, use of population-level screening, ethnic or racial differences, varying exposure to ionizing radiation, and variations in iodine intake (excess or deficiency) $(4,5)$. The rise in thyroid carcinoma incidence, particularly small papillary carcinomas, is largely due to the use of increasingly sensitive detection methods such as high-resolution imaging and ultrasensitive

\footnotetext{
Received Sep. 17, 2020; revision accepted Apr. 14, 2021.

For correspondence or reprints, contact Julie Bolin (julie.bolin@gatewaycc. edu).

Published online July 9, 2021.

COPYRIGHT (C) 2021 by the Society of Nuclear Medicine and Molecular Imaging.
}

thyroglobulin assays, which allow for the detection of previously undetectable thyroid cancers (6-8). Improved technology has allowed for not only an earlier detection of small carcinomas but also a better characterization of the lesions, prompting revisions in clinical management.

\section{THE BASICS}

Thyroid cancer includes 6 main types: papillary, follicular, Hürthle cell, medullary, poorly differentiated, and anaplastic. Clinicians often categorize the 6 main types of thyroid cancer on the basis of the origination cell type (follicular epithelial cell vs. parafollicular C cell) and preservation of cell type (differentiated, poorly differentiated, undifferentiated), as these characteristics relate to diagnosis, prognosis, and treatment (8). Follicular cells, also called thyroid epithelial cells or thyrocytes, line the colloid follicles and concentrate iodine for thyroid hormone synthesis. These cells give rise to papillary, follicular, Hürthle cell, poorly differentiated, and anaplastic thyroid cancers (9). The parafollicular or $\mathrm{C}$ cells are neuroendocrine cells that are scattered throughout the thyroid follicles and are responsible for the synthesis, storage, and secretion of calcitonin. Parafollicular cells give rise to medullary thyroid cancer (9). This continuing education article will focus only on follicular epithelial cell-derived thyroid cancers, which include papillary, follicular, Hürthle cell, poorly differentiated, and anaplastic carcinomas.

\section{PRESERVATION OF CELL TYPE}

Differentiated thyroid cancer represents most (90\%-95\%) types of thyroid cancer and includes papillary, follicular, and Hürthle cell carcinomas (HCCs) (6). Cellular differentiation is a central aspect in the histopathologic classification for thyroid follicular epithelial cell-derived cancers and refers to the process by which a cell becomes specialized to perform a specific function. Differentiated thyroid cancers maintain the characteristics and behavior of normal follicular epithelial cells in thyroid tissue. These cells retain many of the physiologic functions of thyroid cells, including thyroid-stimulating hormone (TSH, also called thyrotropin) stimulation of growth, iodine uptake (expression of sodium iodine symporter), and thyroid hormone production (9). Differentiated thyroid cancers maintain radioactive iodine (RAI) avidity, which permits the use of RAI scintigraphy and treatment as part of the disease management strategy. As a general rule, 
differentiated cancers tend to be less aggressive than undifferentiated cancers (9).

Poorly differentiated thyroid cancer (PDTC) was at one time considered a variant of differentiated thyroid cancer; however, the World Health Organization began recognizing it as a distinct pathologic entity in 2004 because the clinical and histologic features occupy an intermediate position between differentiated thyroid cancer and undifferentiated thyroid cancer (10-13). PDTC occurs de novo; however, there is a theory that PDTC may also transform from differentiated thyroid cancers through the accumulation of genetic abnormalities $(11,14)$. This theory is supported by the frequent cooccurrence of PDTC and differentiated thyroid cancer in the same tumor specimen and the overlap of genetic mutations (11). When PDTC and well-differentiated thyroid cancer are present in the same tissue sample, it is important to note the presence of PDTC, as prognosis and treatment strategies may be guided by the PDTC component (10). Given that PDTCs are often resistant to RAI therapy, these cancers present a therapeutic challenge $(11,12)$. Outcome statistics for PDTCs are worse than for differentiated thyroid cancers. In PDTCs, approximately $50 \%$ of patients survive after $10 \mathrm{y}$, whereas in differentiated thyroid cancers, particularly papillary thyroid carcinoma (PTC), $95 \%$ of patients survive after $10 \mathrm{y}$ (13).

Undifferentiated thyroid cancer includes anaplastic thyroid carcinoma (ATC), a very rare form of thyroid carcinoma accounting for less than $1 \%$ of all thyroid carcinomas (15).
Undifferentiated thyroid cancer may occur de novo or may transform from a previously differentiated thyroid cancer (more commonly PTC, but also follicular thyroid carcinoma [FTC] and PDTC) $(12,14)$. A signature attribute of many advanced tumors is cellular dedifferentiation, whereby cancerous cells maintain little to no resemblance to the normal cells from which the cancer originated. In the context of thyroid cancer, loss of RAI avidity most commonly develops from cellular dedifferentiation, resulting in the impairment of the sodium iodine symporter function $(14,15)$. Loss of iodine avidity excludes the use of RAI scintigraphy and treatment. As thyroid cancer cells dedifferentiate, their glucose metabolism increases, allowing for evaluation with ${ }^{18} \mathrm{~F}$ FDG PET/CT (16). Overall outcomes and survival statistics are poor for undifferentiated thyroid cancer, with a mean survival of $0.5 \mathrm{y}$ after diagnosis (15).

Figure 1 illustrates the cellular differentiation stage for follicular epithelial cell-derived thyroid carcinomas and how it relates to radioiodine scintigraphy/treatment and overall prognosis.

\section{OVERVIEW OF FOLLICULAR EPITHELIAL CELL-DERIVED THYROID CANCERS}

PTC

PTC is the most commonly occurring form of differentiated thyroid cancer and accounts for $80 \%-85 \%$ of all thyroid

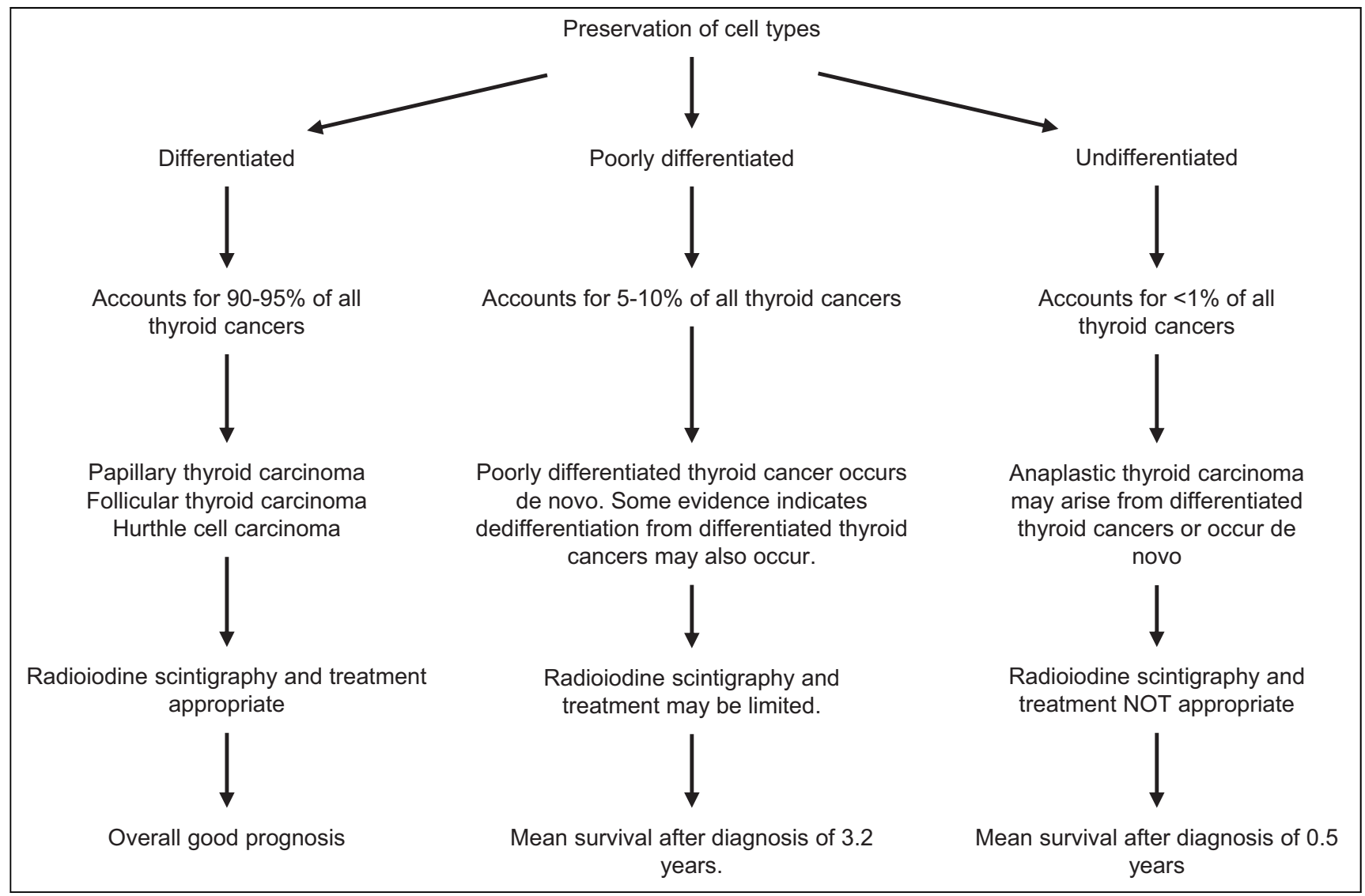

FIGURE 1. Cellular differentiation and diagnostic/prognostic implications. 
cancer (17). Patients are often asymptomatic but may have cervical lymphadenopathy, hoarseness, and dysphagia. Risk factors for PTC include radiation exposure, particularly during childhood, a family history of thyroid malignancy, and inherited conditions such as familial adenomatous polyposis or Cowden syndrome (18). PTC most often affects middleaged women. Thyroid carcinoma accounts for $1.4 \%$ of pediatric malignancies, with PTC being the most common thyroid carcinoma affecting children (3).

In 2017, the World Health Organization recognized 15 histologic subtypes of PTC: conventional/classic, papillary microcarcinoma ( $<1 \mathrm{~cm}$ in diameter), encapsulated, follicular, diffuse sclerosing, tall cell, columnar cell, cribriformmorular, hobnail, PTC with fibromatosis/fasciitislike stroma, oncocytic, solid/trabecular, spindle cell, clear cell, and Warthin-like variant $(12,13)$. Conventional/classic, papillary microcarcinoma, encapsulated, follicular, diffuse sclerosing, and tall cell are the most common and best-documented histologic subtypes $(12,13)$. Because histologic subtypes relate to biologic aggressiveness and prognosis, these distinctions are valuable in terms of disease management strategies and recommendations. The papillary microcarcinoma and encapsulated variants are considered the least biologically aggressive. The tall cell and hobnail variants have the potential to be more aggressive than the conventional/classic variant, and as a result, patients with these tumors may require a more aggressive disease management strategy than do those with conventional/classic papillary cancer of the same stage (12).

In addition to the histologic subtype, PTC tumors range in size and extent of the primary lesion (17). Although there may be microcarcinomas $(<1 \mathrm{~cm}$ in diameter) and intrathyroidal lesions $(>1.5 \mathrm{~cm}$ in diameter) that are confined to the thyroid capsule, it is not uncommon for patients to have multifocal disease, extrathyroidal extension, and cervical lymph node metastasis (17). Up to $27 \%$ of patients have cervical lymph metastasis at the initial presentation (17). Children, more commonly than adults, present with lymph node involvement or metastasis to distant sites such as the lungs (3). Overall, patients with PTC typically have a good prognosis. Increased risk for recurrence or poor prognosis is often related to age (persons $>55$ y old at diagnosis), increased vascular invasion, invasion into the adjacent neck structures, and distant metastasis (17).

\section{FTC}

FTC is the second most common type of thyroid cancer after PTC and accounts for $10 \%$ of differentiated thyroid cancers (19). Unlike PTC, FTC is rarely associated with previous radiation exposure but may be associated with iodine deficiency. FTC typically occurs in a slightly older age group than PTC (40-60 y) and is also less common in children (20). Minimally invasive FTC is limited to microscopic capsular or vascular invasion, whereas widely invasive tumors may have multiple foci and extensive, widespread invasion (21). Given that vascular invasion is common with FTC, metastatic disease may be noted even when the primary thyroid lesion is small. Distant metastatic spread to the lungs or bones is found more commonly with FTC than with PTC (19-21). Poor prognosis is often related to tumor size, degree of vascular and capsular invasion, and age (20).

\section{HCC}

HCC accounts for 3\% of all thyroid cancers. Previously, $\mathrm{HCC}$ was classified as an oxyphilic variant of FTC; however, data from genetic and molecular analysis suggest that HCC is a distinct entity $(22,23)$. Just as with FTC, HCC occurs in a slightly older age group than PTC. Unlike FTC, HCC has more of a propensity to spread to cervical lymph nodes and has a higher risk of distant metastatic disease, particularly skeletal and pulmonary metastasis (24). Another important distinction relevant for patient management is that although metastatic lesions in FTC often concentrate RAI, HCC metastatic foci are often RAI-refractory $(23,25)$.

\section{PDTC}

PDTC has not been studied as commonly as other follicular epithelial cell-derived thyroid cancers, partly because of its overall rarity but also because it was not classified as an independent thyroid cancer histotype until 2004 (11). According to the Turin proposal, the presence of PDTC is determined by the presence of carcinoma of follicular cell derivation, architectural patterns (solid/trabecular/insular architecture), and high-grade features such as nuclear pleomorphism, tumor necrosis, or a high mitotic rate $(11-14)$. PDTC more commonly occurs in women 60-70 y old and is associated with aggressive characteristics such as extrathyroidal extension, regional lymph node metastasis, and distant metastasis. Just as with other forms of follicular epithelial cell-derived thyroid cancers, the most common sites of distant metastasis include the lungs and bones; however, less common metastatic sites also include the liver, skin, ovaries, and retroperitoneal space (11). Although PDTC accounts for only 4\%-7\% of all thyroid cancers, it represents the main cause of death from nonanaplastic follicular cell-derived thyroid cancer (11).

\section{ATC}

ATC is the most aggressive and rarest thyroid cancer. Although ATC accounts for only $1 \%-2 \%$ of all thyroid cancer cases, it is responsible for $14 \%-39 \%$ of deaths related to thyroid malignancy (26). ATC occurs primarily in the geriatric population and is more common in persons 60-70 y old. Patients with ATC often have a large, palpable thyroid mass associated with hoarseness, dyspnea, dysphagia, and vocal cord paralysis (26). The extent of disease may be restricted to the neck region or may include distant metastasis to the lungs, bone, or brain. Although ATC may develop as a singular entity, it may also evolve from or coexist with differentiated thyroid cancer $(12,13,26)$. Given that ATC is a type of undifferentiated thyroid cancer, these cells will not maintain the characteristics and behavior of normal follicular epithelial cells and thus will not concentrate RAI. 


\section{GENETIC CHANGES INVOLVED IN THYROID CARCINOGENESIS}

Table 1 defines abbreviations used in discussing the genetics of thyroid carcinogenesis.

\section{MAPK Pathway}

In recent years, there have been multiple studies evaluating the genetic alterations and molecular mechanisms underlying thyroid carcinogenesis and their prevalence in follicular epithelial cell-derived thyroid carcinomas. An understanding of these mutations may explain the diverse clinical characteristics of thyroid follicular epithelial cell-derived carcinomas and provide diagnostic information relative to treatment. Mutations in both oncogenes and tumor suppressor genes acquired along the path of tumor progression allow cancerous cells to evade the normal control of cell cycle and apoptotic processes and promote the transition from localized to metastatic disease. Oncogenic activation of the mitogen MAPK is considered the most common molecular alteration in thyroid cancer and is believed to initiate carcinogenesis (6). The MAPK pathway is a key molecular signaling pathway responsible for the regulation of several diverse cellular functions, including cellular growth, proliferation, and angiogenesis. In the normally functioning cell, the MAPK pathway is triggered by the binding and activation of receptor tyrosine kinases, which transmit growth signals from the plasma membrane to the nucleus (6). Oncogenic alterations in the MAPK pathway influence processes that are crucial for cancer development and progression. In oncogenic cells, the MAPK pathway is driven by mutations including RET/PTC, RAS, and $B R A F(6)$. These driver mutations are initiating mutations and occur early in thyroid carcinogenesis (14).

\section{RET/PTC Rearrangements}

$R E T$ protooncogene rearrangements are most commonly found in PTC (termed RET/PTC rearrangements) and lead to the constitutive activation of the RET tyrosine kinase domain and subsequent activation of the MAPK signaling pathway (27). RET/PTC rearrangements are found in $20 \%-70 \%$ of PTCs and are more commonly associated with pediatric thyroid cancers (27). Although 13 RET/PTC rearrangements have been found, RET/PTC1 and RET/PTC3 are the most

TABLE 1

Genetic Abbreviations

\begin{tabular}{lc}
\hline Name & Abbreviation \\
\hline Mitogen-activated protein kinase & MAPK \\
$\begin{array}{l}\text { Rearrangement during transfection/ } \\
\text { papillary thyroid cancer mutations }\end{array}$ & RET/PTC \\
Rat sarcoma point mutations & RAS \\
B-rapidly accelerated fibrosarcoma & BRAF \\
$\begin{array}{l}\text { Telomerase reverse transcriptase } \\
\text { promoter }\end{array}$ & TERT \\
Tumor protein & Tp53 \\
$\begin{array}{l}\text { Phosphatase and tensin homolog } \\
\text { deleted from chromosome 10 }\end{array}$ & PTEN \\
\hline
\end{tabular}

common. RET/PTC1 is more prevalent in sporadic PTC, and $R E T / P T C 3$ is highly prevalent in radiation-induced PTC. RET/PTC rearrangements are also found in the follicular variant of PTC and in FTC (15). The prognostic significance of RET/PTC rearrangements is not fully established. RET/PTC1 does not correlate with clinical pathologic features of PTC; however, RET/PTC3 is associated with aggressive characteristics and poor prognostic factors, including greater primary tumor size, cellular variations, and a more advanced stage at diagnosis $(15,27)$.

\section{RAS Mutations}

The $R A S$ oncogene mutations play an important role in thyroid oncogenesis through the regulation of 2 important signaling pathways: the MAPK cascade, which is responsible for cellular proliferation, and the phosphatidylinositol 3-kinase $(\mathrm{PI} 3 \mathrm{~K}) /$ protein kinase B (AKT) pathway, which is important

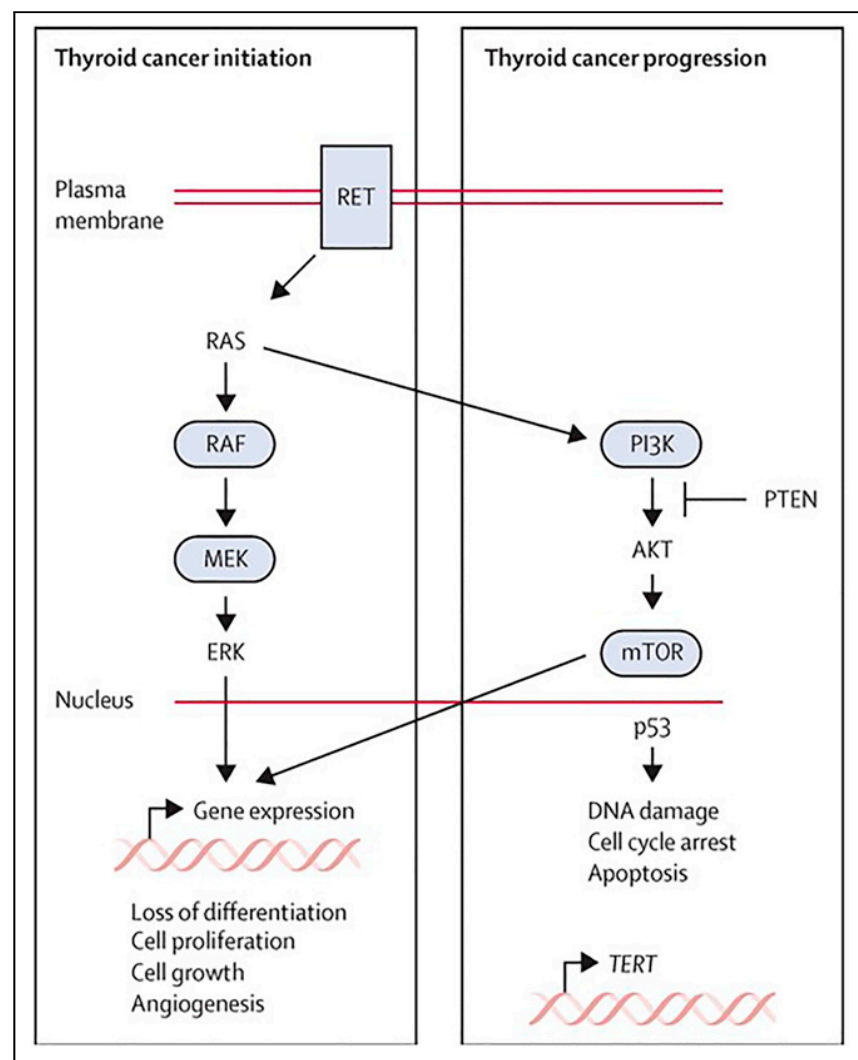

FIGURE 2. Key molecular signaling pathways involved in thyroid cancer initiation and progression. (Left) MAPK pathway, which is activated by mutations in RET, RAS, and BRAF. (Right) Pathways involved in tumor progression, including PI3K/AKT, p53 tumor suppressor, and TERT. Blue boxes represent molecular targets for therapies approved by Food and Drug Administration. ERK = extracellular-signal-regulated kinase; MEK = mitogen/extracellular-signal-regulated kinase; mTOR = mammalian target of rapamycin; PTEN = phosphatase and tensin homolog mutation; p53 $=\mathrm{Tp} 53$ or tumor protein; RAF = rapidly accelerated fibrosarcoma; RAS = rat sarcoma point mutations; RET = rearrangement during transfection; TERT = telomerase reverse transcriptase. (Reprinted with permission of (6).) 
for cell survival (Fig. 2) (6,15). Oncogene activation in the 3 $R A S$ protooncogenes (HRAS, KRAS, and NRAS) is found in most human cancers, including thyroid cancer. $R A S$ mutations occur in almost every type of thyroid cancer and in benign follicular adenomas: $20 \%-25 \%$ of benign follicular adenomas, $10 \%-20 \%$ of PTCs, $30 \%-45 \%$ of follicularvariant papillary thyroid cancers, $30 \%-35 \%$ of FTCs, $20 \%-40 \%$ of PDTCs, and 10\%-20\% anaplastic thyroid cancers (ATCs) (9,28). RAS mutation alone is most likely associated with limited aggressiveness of thyroid cancer; however, $R A S$ may coexist with other mutations such as TERT or $P T E N$, both of which have been implicated in aggressive clinicopathologic behavior $(28,29)$.

\section{BRAF Mutations}

Although more than 40 point mutations have been documented in $B R A F$, the most frequent mutation is $T 1799 A$ $B R A F$, resulting in $\mathrm{V} 600 \mathrm{E}$ protein kinase (6). BRAF V600E is exclusive to PTC, PTC-derived PDTC, and ATC. The BRAF V600E mutation occurs in about $40 \%-45 \%$ of all PTCs and in about $60 \%$ of BRAF-associated PTCs (30). $B R A F$ V600E mutation is noted in the potentially more aggressive PTC variants, including multinodular/diffuse forms of the follicular variant of PTC (15). BRAF V600E mutation causes constitutive activation of the BRAF kinase and MAPK signaling pathway and has been associated with aggressive clinicopathologic features such as extrathyroidal invasion, lymph node metastasis, vascular invasion, and a primary tumor of advanced stage (15). In $B R A F$-associated PTC, both vascular endothelial growth factor and platelet-derived growth factor are upregulated $(31,32)$. Vascular endothelial growth factor and platelet-derived growth factor are potent stimulators of angiogenesis, a process that is vital to carcinogenesis. $B R A F$ mutations have also been implicated in the loss of cellular differentiation resulting in decreased expression of sodium iodide symporter, TSH receptor, and thyroglobulin, thereby creating a situation for iodine-refractory disease $(6,9,14)$. Although $B R A F$ mutations have been implicated in aggressive behavior, there is no clear consensus. Frequently, classic PTC and papillary microcarcinomas have associated $B R A F$ mutations. Despite these mutations, classic PTC and papillary microcarcinomas maintain an excellent prognosis. Isolated $B R A F$ mutations may not substantially contribute to risk stratification and increased specific disease mortality. Cancer evolution is triggered by the sequential accumulation of mutations that bestow metastatic potential. $B R A F$ mutations are frequently associated with TERT mutations. Thyroid cancers displaying both BRAF V600E and TERT mutations exhibit more aggressive clinicopathologic behavior than do thyroid cancers displaying only $B R A F$ mutations (29).

\section{NON-MAPK GENETIC MUTATIONS}

\section{TERT Mutations}

A genetic alteration in TERT has been associated with increased aggressiveness and poor patient prognosis.
Telomerase is the enzyme involved in telomere elongation, whose main function is preservation of chromosome integrity and genomic stability. Telomeres are repetitive DNA sequences at the ends of chromosomes acting as protective caps. Telomeres are continually shortened with each successive generation. When telomeres reach a critically short length, the chromosomes participate in breakage-fusion-breakage cycles resulting in cell crisis and apoptosis (29). Therefore, telomeres allow a cell to replicate only a certain number of times. Since cancer cells seek to grow at an unconstrained rate, the shortened telomeres and subsequently restricted replication present a significant problem. In cancer cells, telomere length is maintained by telomerase, which functions to elongate telomeric DNA by using its 2 components: an RNA template and a reverse transcriptase (29). Mutations in TERT are sufficient to restore the activity of the telomerase complex (15). Most adult differentiated cells do not express or have very low expression of telomerase; however, telomerase is significantly expressed in $90 \%$ of human cancers, including differentiated, poorly differentiated, and undifferentiated thyroid cancers (29). By expressing higher levels of reverse transcriptase, and therefore allowing for increased telomerase activity, carcinoma cells prevent critically short telomeres and apoptosis, meaning the oncogenic changes bestow an extended life or immortality.

Independent of its function in telomere length maintenance, TERT mutations have also been associated with metastatic capability and cellular dedifferentiation $(15,29)$. Studies have indicated that TERT may encourage metastasis through the activation of the epithelial-mesenchymal transition. This transition is a process by which epithelial cells lose their normal characteristics such as intercellular adhesion and lack of motility and acquire mesenchymal cell properties such as reduced intercellular adhesion, increased motility, invasiveness, and apoptosis resistance (29). Activation of the epithelial-mesenchymal transition process is triggered by various stimuli resulting in the silencing of intracellular adhesion molecules such as epithelial cadherin and the upregulation genes promoting the mesenchymal shift (29). The epithelial-mesenchymal transition is also suggested to promote the dedifferentiation process, resulting in poorly differentiated or undifferentiated cancers (29).

\section{Tp53 Mutation}

Alterations in several tumor suppressor genes have been associated with thyroid oncogenesis, but the most widely studied is the Tp53 gene. p53 (also known as Tp53) is a key tumor suppressor protein known as the "guardian of the genome" because of its functions in maintaining genomic integrity through control of cell division, apoptosis, DNA repair, and angiogenesis (15). Cancer cells with a Tp53 mutation have evolved the ability to bypass the cell cycle checkpoints, to favor antiapoptotic pathways, and to proliferate uncontrollably. The amount of p53 in normal cells is maintained at a very low level; however, Tp53 mutations and p53 overexpression are frequent in several human cancers and occur in well-differentiated thyroid cancers $(40 \%$ of 
PTCs and 22\% of oncocytic FTCs), as well as in a high proportion of PDTC and ATCs (particularly those originating from PTC), which indicates that $T p 53$ mutations promote the transition from differentiated to undifferentiated thyroid cancer (15). Tp 53 mutations are most noted in the later stages of oncogenesis and occur simultaneously with a substantial increase in cellular proliferation (15). Tp53 mutations have been noted in cancers with BRAF mutations and RET/PTC rearrangements and are believed to promote extrathyroidal extension and distant metastasis (15).

Figure 2 illustrates key molecular signaling pathways involved in thyroid cancer initiation and progression. Table 2 reviews genetic mutations associated with thyroid carcinogenesis.

\section{ASSESSMENT AND TREATMENT}

Thyroid cancers of follicular epithelial cell origin exhibit highly variable clinical manifestations ranging from microcarcinomas with very low tumor-specific morbidity or mortality to very aggressive thyroid carcinomas such as anaplastic carcinoma. Diagnostic workup and standard treatments such as the use of surgery, RAI therapy, and TSH suppression therapy are individualized to the patient and the clinical objectives. Despite the increased incidence of thyroid carcinoma in the past 30 years, there has not been a substantial increase in mortality $(6,7,33)$. This has sparked a discussion in the medical community about the potential for overdiagnosis and overtreatment. In 2015, American Thyroid Association (ATA) guidelines for medical management of adults with thyroid nodules and differentiated thyroid cancer emphasized a distinction between low-risk and high-risk patient groups, with physicians identifying patients who require more aggressive treatment while sparing patients with a low risk from unnecessary diagnostic procedures and treatments $(33,34)$. The 2015 ATA guidelines are not inclusive of all proper approaches or methods and were met with controversy by the medical community. In 2018, the Martinique Working Group, comprising representatives from the ATA, the European Association of Nuclear Medicine, the European Thyroid Association, and the Society of Nuclear Medicine and Molecular Imaging, published an authoritative report called "The Martinique Principles," which highlighted controversies in the medical management of thyroid cancer (35). Although the current article will not highlight the spectrum of expert opinions presented by members of the Martinique Working Group, it should be stated that there is still debate within the medical community regarding disease management strategies.

Treatment decisions for patients with differentiated thyroid cancer are based on strategies for risk assessment that consider symptom manifestation, histology, tumor size, tumor invasiveness, lymph node involvement, distant metastasis, and diagnostic imaging. Cervical lymph node sonography, which provides information on the size, number, and location of thyroid lesions and on vascularity, lymph node involvement, and adjacent tissue invasion, is widely used as the primary imaging tool when determining surgery and treatment options (6). The 2015 ATA guidelines recommend preoperative sonography for patients undergoing surgery for confirmed or suspected thyroid malignancy $(33,34)$. Preoperative

TABLE 2

Genetic Mutations and Associated Prognostic Significance

\begin{tabular}{|c|c|c|}
\hline Genetic mutation & Cancers displaying genetic mutation & Prognostic significance \\
\hline$B R A F$ & PTC (BRAF V660E), PDTC, ATC & $\begin{array}{l}\text { No clear consensus on prognostic significance; } \\
\text { linked to extrathyroidal invasion, lymph node } \\
\text { metastasis, vascular invasion, advanced tumor } \\
\text { stage in primary tumor, and multifocality; linked to } \\
\text { cellular dedifferentiation; linked to loss of iodine } \\
\text { avidity; upregulates platelet-derived growth factor; } \\
\text { upregulates vascular endothelial growth factor }\end{array}$ \\
\hline PTEN & Differentiated thyroid cancers, PDTC & Uncontrolled cell growth and proliferation \\
\hline RAS & PTC, PTC-FV, FTC, PDTC, ATC & $\begin{array}{c}\text { RAS mutations alone likely associated with limited } \\
\text { aggressiveness }\end{array}$ \\
\hline$R E T / P T C$ rearrangements & PTC (adult and pediatric), PTC-FV, FTC & $\begin{array}{l}\text { Not fully established; RET/PTC1 does not correlate } \\
\text { with clinical pathologic features; RET/PTC3 is } \\
\text { associated greater primary tumor size, cellular } \\
\text { variations, and more advanced stage at diagnosis }\end{array}$ \\
\hline TERT & $\begin{array}{l}90 \% \text { of human cancers, differentiated } \\
\text { thyroid cancers, PDTC, ATC }\end{array}$ & $\begin{array}{l}\text { Restores telomerase complex activity (prevents } \\
\text { apoptosis); promotes epithelial mesenchymal } \\
\text { transition, which promotes metastasis and cellular } \\
\text { dedifferentiation }\end{array}$ \\
\hline Tp53 & PTC, FTC, PDTC, ATC & $\begin{array}{l}\text { Extrathyroidal extension; distant metastasis; } \\
\text { potentially promotes cellular dedifferentiation }\end{array}$ \\
\hline
\end{tabular}

$B R A F=$ B-rapidly accelerated fibrosarcoma mutation; PTEN = phosphatase and tensin homolog mutation; $R A S=$ rat sarcoma point mutation; PTC-FV = follicular variant of papillary thyroid cancer; $R E T / P T C=$ rearrangement during transfection/papillary thyroid cancer (RET/ PTC) mutation; TERT = telomerase reverse transcriptase mutation. 
cervical lymph node sonography identifies $20 \%-30 \%$ of suggestive lymph nodes; identifying such nodes may change the surgical plan and allow for a more complete initial surgical dissection $(6,33)$. For patients with papillary microcarcinomas $1 \mathrm{~cm}$ or smaller and with no evidence of lymph node metastasis, nonsurgical management may be possible $(6,7,34)$. If surgery is indicated, a hemithyroidectomy is potentially an option for unifocal tumors smaller than $4 \mathrm{~cm}$ and with no evidence of lymph node metastasis or invasion into adjacent neck structures; however, a total thyroidectomy may be indicated if the disease management team believes RAI therapy will be used or if structural changes dictate surgical preferences $(6,7,33)$.

In addition to sonography, the 2015 ATA guidelines advocate for the presurgical use of cross-sectional imaging with $\mathrm{CT}$ and MRI when there is sonographic evidence of extrathyroidal extension, significant cervical lymphadenopathy, tumor adherence to neck structures, or patient symptoms such as hoarseness and dysphagia, as these situations are potentially high-risk $(33,34)$. Use of iodinated contrast medium in these patients may delay RAI imaging and therapy for several months; however, the complete surgical excision of all thyroid tissue and locoregional metastasis is a critical element in patient management (33). Rarely, additional preoperative imaging is required for differentiated thyroid cancer, unless there is suspicion of widespread or distant metastasis. ${ }^{18} \mathrm{~F}$ FDG PET/CT may be indicated for initial staging in these cases but is more commonly used for postsurgical evaluation in RAI-refractory disease (5).

After surgery, the disease management team will determine the need for RAI ablation, TSH suppression therapy, or both. The 2015 ATA guidelines identify high, intermediate, and low risk for thyroid cancer recurrence and include clinical symptoms, postsurgical thyroglobulin levels, and pathologic features in the determination of risk of recurrence $(6,34)$. The extent of surgery will influence the acceptable ranges for thyroglobulin, as patients who had a hemithyroidectomy will have a higher thyroglobulin level because of the remaining thyroid tissue, which is likely benign (7).

\section{TSH Suppression Therapy}

In patients who have had a partial or total thyroidectomy, thyroid hormone replacement may be used not only to replace endogenous thyroid hormone but also to inhibit tumor progression or recurrence because TSH encourages cellular proliferation in differentiated thyroid cancer cells. TSH suppression therapy significantly reduces recurrence and thyroid cancer-related mortality $(6,36)$. The goal of TSH suppression therapy is to reduce TSH to below the reference range (0.4-4.5 mIU/L). The appropriate TSH concentration as determined by the disease management team may vary among patients given the extent of surgery and the distinction between low-risk and high-risk status. Reducing TSH concentrations to $0.1 \mathrm{mIU} / \mathrm{L}$ can improve outcomes for highrisk patients; however, this reduction requires doses of thyroid hormone replacement that may induce subclinical hyperthyroidism (6). Potential adverse effects include osteoporosis in postmenopausal women, angina, and atrial fibrillation (6). In patients with low-risk thyroid cancer, a more conservative approach with the goal of thyroid hormone replacement therapy to maintain TSH at or slightly below the lower limit of normal $(0.1-0.5 \mathrm{mIU} / \mathrm{L})$ may be appropriate (36). Patients who undergo a lobectomy instead of total thyroidectomy may not require TSH suppression therapy if their TSH serum levels are maintained at the lower limit of normal $(0.5-2.0 \mathrm{mIU} / \mathrm{L})(36)$.

Figure 3 illustrates the negative-feedback loop for thyroid hormone synthesis. The hypothalamic-pituitary axis regulates TSH release through a negative-feedback loop. TSHreleasing hormone stimulates anterior pituitary thyrotrophs to secrete TSH. When TSH binds to the TSH receptor on thyroid follicular cells, it stimulates iodine uptake, thyroid hormone (triiodothyronine, tetraiodothyronine) secretion, and thyroid gland growth and differentiation. Of the thyroid hormones released, approximately $80 \%$ are in the form of tetraiodothyronine and $20 \%$ in the form of triiodothyronine (37). Triiodothyronine has a short half-life but is most active on the nuclear receptor, whereas tetraiodothyronine has a long half-life but is less active in binding to the nuclear receptor. Because tetraiodothyronine becomes deiodinated and converted into triiodothyronine by most tissues (especially the liver and kidneys), tetraiodothyronine acts as a reservoir for triiodothyronine (37). Serum levels of thyroid hormone exert negative-feedback control on TSH-releasing hormone and TSH. High thyroid hormone levels decrease TSH secretion, whereas low thyroid hormone levels stimulate TSH synthesis

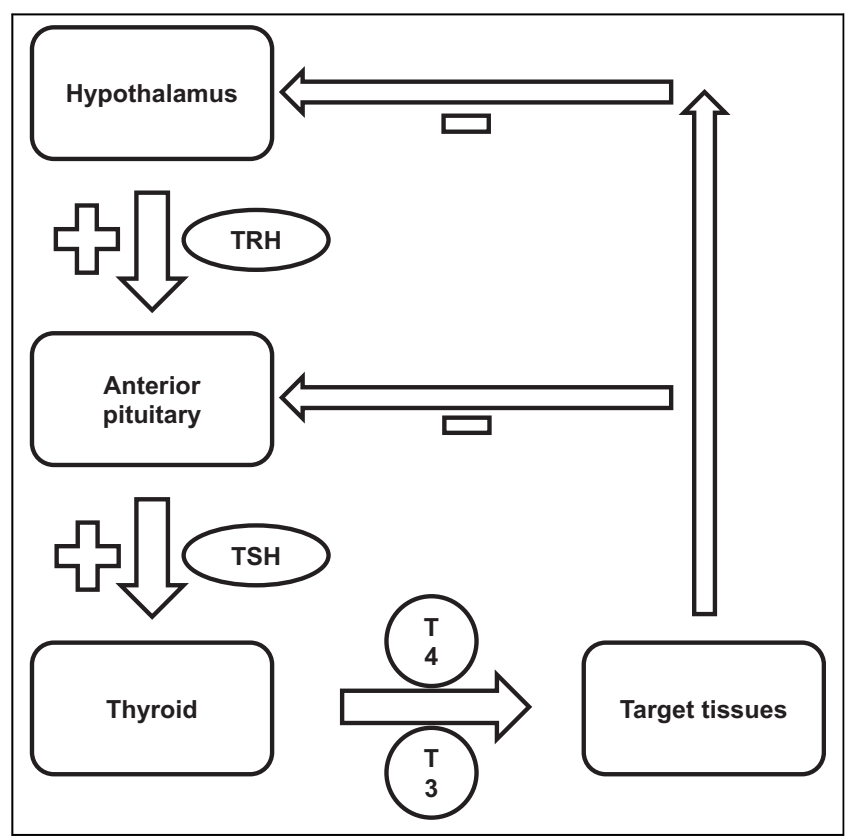

FIGURE 3. Thyroid hormone synthesis negative-feedback loop. Arrow with plus sign indicates stimulation. Arrow with minus sign indicates inhibition. T3 = triiodothyronine; T4 = tetraiodothyronine; $\mathrm{TRH}=\mathrm{TSH}$-releasing hormone. 
and secretion. As this relates to thyroid cancer, patients who have had a total thyroidectomy require TSH suppression therapy for 2 reasons: to replace endogenous thyroid hormone and to suppress TSH. TSH suppression is an important aspect of treatment because TSH may stimulate cellular proliferation in differentiated thyroid cancer cells.

\section{RAl Therapy}

In previous years, many clinicians routinely recommended RAI therapy for all patients with differentiated thyroid cancer, except in patients with papillary microcarcinoma. However, the 2015 ATA guidelines proposed a more conservative approach based on specific histopathologic features that could modulate risk of recurrence or disease-specific mortality, disease follow-up implications, potential adverse effects, and physician and patient preferences $(6,7,34)$. The goals of RAI therapy are remnant ablation, adjuvant therapy, and treatment of known residual or recurrent disease.

Remnant ablation eliminates residual clusters of presumably normal or benign thyroid tissue, which incorporates iodine and produces thyroglobulin, thereby complicating efforts to use thyroglobulin serum assays and RAI scintigraphy to identify persistent disease or recurrence (6). Potential benefits to remnant ablation include improved initial staging and the clinical use of RAI whole-body scintigraphy and thyroglobulin serum assays as a follow-up for cancer recurrence. However, use of RAI whole-body scintigraphy is not used as common as it once was because of the increased sensitivity of cervical lymph node sonography ( 6 ), which is cheaper by comparison, is more widely available, has no associated radiation exposure, has no potential side effects, and does not require discontinuation of thyroid hormone replacement therapy or use of $\mathrm{TSH} \alpha$ injections (6). Cervical lymph node sonography combined with postoperative serum thyroglobulin (either with the patient taking thyroid hormone replacement or after TSH stimulation) are very sensitive methods for detecting persistent disease or a thyroid remnant, for predicting the potential for disease recurrence, and for tailoring future diagnostic and therapeutic approaches (6,33). Postoperative whole-body RAI scintigraphy may still be indicated when the extent of a thyroid remnant or residual disease cannot be accurately determined from cervical lymph node sonography and surgical reports or when the results of whole-body RAI scintigraphy may alter treatment decisions. If remnant ablation is deemed appropriate for low- to intermediate-risk patients, lower dose ranges for ${ }^{131} \mathrm{I} \mathrm{NaI}$ $(1,110 \mathrm{MBq}$, or $30 \mathrm{mCi})$ may be preferred over high doses, depending on the size of the remnant $(7,33)$.

Use of RAI for initial adjuvant therapy destroys microscopic foci that may or may not be present in the thyroid remnant or elsewhere in the body after surgical resection. The goals of adjuvant therapy are to reduce the risk of disease recurrence and improve long-term outcomes. Given that patients are selected for adjuvant therapy with regard to risk of recurrence instead of known disease, some patients who receive adjuvant therapy may have been adequately treated by their primary surgery (35). For patient selection and RAI dose determination, clinicians evaluate several factors, such as the likelihood of improved clinical outcomes, potential side effects, the results of postsurgical follow-up RAI scintigraphy, thyroglobulin assays, the extent of thyroid surgery, and the preferences of the patient and disease management team (35). If RAI is indicated as adjuvant therapy for patients without known distant metastasis, a dose range of up to 5,550 $\mathrm{MBq}(150 \mathrm{mCi})$ is generally recommended but should be tailored to the specific patient on the basis of individual risk, the lowest activity needed for effective treatment, and specific recommendations from the disease management team (33).

The third use of RAI therapy is for patients with known iodine-avid metastatic disease or for recurrence. Typical doses are 3,700-7,400 MBq (100-200 $\mathrm{mCi})$ and may be based on the observations and experience of the disease management team or estimated by dosimetry, with a goal of limiting excess radiation exposure to the bone marrow and avoiding hematopoietic toxicities $(7,33)$. Just as with adjuvant RAI therapy, there may be multiple factors that account for the determined dose. Dose-related impairment of testicular function and transient ovarian failure have been observed after RAI therapy. General recommendations include avoidance of pregnancy for at least 6 mo after treatment, or longer if additional RAI therapy may be indicated. For men who may receive multiple RAI treatments, consideration of sperm banking may be necessary if there is an anticipated cumulative ${ }^{131} \mathrm{I}$-sodium iodide dose greater than $14 \mathrm{GBq}$ (378 mCi) (38).

For patients with iodine-refractory disease, RAI therapy is less likely to provide benefit. The Martinique Working Group described 5 common clinical scenarios that may indicate an iodine-refractory state: no RAI uptake on a diagnostic whole-body scan, no RAI uptake on a scan obtained several days after RAI therapy administration, lack of RAI uptake in all tumor foci, progression of differentiated thyroid cancer metastasis despite RAI uptake, and progression of differentiated thyroid cancer metastasis despite cumulative ${ }^{131} \mathrm{I} \mathrm{NaI}$ doses of more than 22.2 GBq (600 mCi) (35). Potential treatments for iodine-refractory disease include external radiation therapy, chemotherapy, and targeted molecular therapy.

\section{Targeted Molecular Therapy for Advanced Differentiated Thyroid Cancer}

Table 3 defines abbreviations used in discussions of targeted molecular therapy for advanced differentiated thyroid cancer.

Both papillary and follicular carcinomas are welldifferentiated thyroid cancers and are considered highly treatable by conventional treatments, which include surgery, RAI ablation, and TSH suppression therapy. Although HCC is also considered differentiated and may also be treated with conventional treatments, it may prove more RAI-resistant than PTC and FTC. A small percentage of patients with differentiated thyroid cancer develop iodine-refractory metastatic disease, thereby limiting treatment options (39). Although 
TABLE 3

Targets in Molecular Therapy

\begin{tabular}{ll}
\hline Abbreviation & Definition \\
\hline FGF & Fibroblast growth factor \\
FLT-3 & $\begin{array}{c}\text { Type III receptor tyrosine kinase } \\
\text { KIT }\end{array}$ \\
$\begin{array}{c}\text { Type of receptor tyrosine kinase and type of } \\
\text { tumor marker }\end{array}$ \\
MEK & Mitogen/extracellular signal-regulated kinase \\
mTOR & Mammalian target of rapamycin \\
PDGF $\alpha$ & Platelet-derived growth factor $\alpha$ \\
PDGFR $\beta$ & Platelet-derived growth factor receptor $\beta$ \\
PPAR $\gamma$ & Peroxisome proliferator-activated receptor $\gamma$ \\
RET & Rearrangement during transfection \\
RET/PTC & Rearrangement during transfection/papillary \\
& thyroid cancer \\
VEGFR & Vascular endothelial growth factor receptor \\
\hline
\end{tabular}

conventional treatments may also be used in PDTC, many cases are RAI-resistant (14). Thyroid cancers that are no longer responsive to RAI therapy are still candidates for other forms of treatment, including standard chemotherapy and targeted therapy. Targeted therapy and chemotherapy differ in their mechanism of cancer treatment. Targeted drug therapy specifically prohibits the action of key proteins involved in cancer-specific activities, whereas traditional chemotherapy affects all rapidly proliferating cells, including normal cells. Targeted drug therapy is an active area of cancer research, and currently there are 2 approved drugs that target the MAPK cascade. The U.S. Food and Drug Administration approved 2 multikinase inhibitors for the treatment of advanced differentiated thyroid cancer refractory to RAI treatment: sorafenib (approved in 2013) and lenvatinib (approved in 2015). Multikinase inhibitors block the effect of tyrosine kinases that are overactive in the MAPK pathways. Sorafenib is a kinase inhibitor that inhibits multiple intracellular and cell-surface kinases (KIT, FLT-3, RET, RET/PTC, VEGFR 1-3, and PDGFR- $\beta$ ) (40) that are implicated in tumor cell signaling, angiogenesis, and apoptosis. Lenvatinib is also a kinase inhibitor that inhibits the vascular endothelial growth factor receptors as well as other kinases (FGF receptors, PDGF $\alpha$, KIT, and RET) associated with pathogenic angiogenesis, tumor growth, and cancer progression $(39,41)$. In addition to the multikinase inhibitors, selective kinase inhibitors are also therapeutic options for advanced differentiated thyroid carcinoma. In May 2020, the Food and Drug Administration approved a highly selective kinase inhibitor, selpercatinib, for the treatment of iodine-refractory $R E T$-positive advanced thyroid cancer $(39,42)$.

A promising use of kinase inhibitors in iodine-refractory differentiated thyroid cancer is the resensitization to RAI. BRAF V600E mutations coincide with reduced sodium iodine symporter expression, thereby inhibiting RAI uptake (39). In a study conducted by Rothenberg et al., 10 patients with iodine-refractory thyroid cancer received a $B R A F$ inhibitor for $25 \mathrm{~d}$. Six of the 10 patients demonstrated uptake of RAI and could be retreated with RAI $(39,43)$. RAI resensitization has also been documented in a phase II trial with an MEK inhibitor, selumetinib, in patients expressing $B R A F$ or $R A S$ mutation (39). Figure 2 illustrates the key molecular signaling pathways involved in thyroid cancer initiation and progression. MEK is a downstream protein in the RAS/RAF/MEK MAPK signaling cascade. Selumetinib inhibits overstimulation of this pathway. Of the 20 patients in the trial ( 9 with $B R A F$ mutation and 5 with NRAS mutation), 12 demonstrated RAI uptake in response to selumetinib use. Eight of the 12 patients could be treated with RAI. Of the 8 patients who received another RAI treatment, 5 had NRAS mutations and one had a BRAF mutation (39).

\section{Treatment for Undifferentiated Thyroid Carcinoma}

Anaplastic thyroid cancer is a diagnostic and therapeutic challenge because it is very rare, advances quickly, and is not iodine-avid, which prevents the use of RAI for evaluation and treatment. In these cases, ${ }^{18} \mathrm{~F}$-FDG PET is useful for evaluation and follow-up after treatments. Initial treatment includes surgical resection and airway management (often a tracheostomy) followed by external-beam radiation therapy with or without use of doxorubicin, taxanes, or cisplatin $(6,39,44)$. Patients who have unresectable cancer but without distant metastasis are generally treated with palliative chemoradiation (6). Genetic marker analysis has led to the development of therapeutic agents that target specific molecular pathways associated with ATC. For example, clinical trials with $B R A F$ inhibitors (e.g., dabrafenib), MEK inhibitors (e.g., trametinib and lenvatinib), mTOR inhibitors, or PPAR $\gamma$ inhibitors may be appropriate for patients with $\operatorname{ATC}(39,44)$. MEK is a key protein in the MAPK signaling cascade, whereas mTOR is a key protein in the PI3K-AKT pathway. PPAR $\gamma$ acts as a tumor suppressor gene, upregulating important enzymes that control the cell cycle $(6,44)$.

\section{CONCLUSION}

Thorough evaluation of decades' worth of data on thyroid carcinoma incidence, treatment, and outcomes have called into question the most appropriate management of patients with follicular epithelial cell-derived thyroid carcinoma. The 2015 ATA guidelines called for a more conservative approach for low-risk malignancies but take into account physician and patient preferences. However, given the lack of consensus on the recommendations for a reduction in the extent of surgery and RAI therapy, the preferences of the disease management team may vary among institutions. The controversy regarding the 2015 ATA guidelines for diagnostic and therapeutic use of RAI prompted a collegial discussion among members of the greater nuclear medicine community and resulted in an authoritative paper, "The Martinique Principles." These principles helped to define common terminology for the goals of RAI therapy (ablation, adjuvant treatment, or treatment of known residual or recurrent disease), reiterated the importance of evaluating multiple factors when determining the appropriateness of RAI therapy, and 
acknowledged the limitations of current scientific literature. Most importantly, the Martinique Working Group established a platform for evaluating current practices, exchanging ideas, and critiquing the literature that will help guide future research and optimize thyroid cancer diagnosis and management.

Genetic and molecular evaluation of thyroid carcinoma has identified 2 key signaling pathways primarily involved in carcinogenesis and oncogenesis. The MAPK pathway is implicated in loss of differentiation, cell growth, cell proliferation, and angiogenesis, whereas the PI3K-AKT pathway promotes tumor progression. Many genetic alterations coexist, and as these mutations accumulate, the MAPK and PI3K-AKT pathways are activated. The use of molecular analysis to classify malignancies and predict tumor progression will evolve as more molecular markers are developed. This advancement will likely lead to new prognostic tools and potential therapeutic agents for improved patient management and treatment strategies.

\section{DISCLOSURE}

No potential conflict of interest relevant to this article was reported.

\section{REFERENCES}

1. Cancer stat facts: thyroid cancer. Surveillance Epidemiology, and End Results website. https://seer.cancer.gov/statfacts/html/thyro.html. Accessed June 30, 2021.

2. Thyroid cancer risk factors. American Cancer Society website. https://www.cancer. org/cancer/thyroid-cancer/causes-risks-prevention/risk-factors.html. Accessed June 30, 2021.

3. Verburg FA, Van Santen HM, Luster M. Pediatric papillary thyroid cancer: current management challenges. Onco Targets Ther. 2016;10:165-175.

4. Salehiniya A, Pakzad R, Hassanipour S, Mohammadian M. The incidence and mortality of thyroid cancer and its relationship with HDI in the world. World Cancer Res J. 2018;5:e1091.

5. Lubitz CC, Sosa JA. The changing landscape of papillary thyroid cancer: epidemiology, management, and the implications for patients. Cancer. 2016;122:3754-3759.

6. Cabanillas ME, McFadden DG, Durante C. Thyroid cancer. Lancet. 2016;388:27832795 .

7. Nabhan F, Ringel MD. Thyroid nodules and cancer management guidelines: comparisons and controversies. Endocr Relat Cancer. 2017;24:R13-R26.

8. What is thyroid cancer? American Cancer Society website. https://www.cancer.org/ cancer/thyroid-cancer/about/what-is-thyroid-cancer.html. Accessed June 30, 2021.

9. Younis E. Oncogenesis of thyroid cancer. Asian Pac J Cancer Prev. 2017;18:11911199.

10. Cherkaoui GS, Guensi A, Taleb S, et al. Poorly differentiated thyroid carcinoma: a retrospective clinicopathological study. Pan Afr Med J. 2015;21:137.

11. Landa I, Ibrahimpasic T, Boucai L, et al. Genomic and transcriptomic hallmarks of poorly differentiated and anaplastic thyroid cancers. J Clin Invest. 2016;126:1052-1066.

12. Lam AK. Pathology of endocrine tumors update: World Health Organization new classification 2017-other thyroid tumors. AJSP: Reviews \& Reports. 2017;22: 209-221.

13. Kakudo K, Bychkov A, Bai Y, et al. The new 4th edition World Health Organization classification for thyroid tumors, Asian perspectives. Pathol Int. 2018;68:641-664.

14. Soares P, Lima J, Preto A, et al. Genetic alterations in poorly differentiated and undifferentiated thyroid carcinomas. Curr Genomics. 2011;12:609-617.

15. Penna GC, Vaisman F, Vaisman M, Sobrinho-Simões M, Soares P. Molecular markers involved in tumorigenesis of thyroid carcinoma: focus on aggressive histotypes. Cytogenet Genome Res. 2016;150:194-207.

16. Prante O, Maschauer S, Fremont V, et al. Regulation of uptake of ${ }^{18}$ F-FDG by a follicular human thyroid cancer cell line with mutation-activated K-Ras. J Nucl Med. 2009;50:1364-1370.

17. Limaiem F, Rehman A, Mazzoni T. Papillary thyroid carcinoma. National Center for Biotechnology Information website. https://www.ncbi.nlm.nih.gov/books/ NBK536943/. Published January 6, 2021. Accessed June 30, 2021.
18. Schmidbauer B, Menhart K, Hellwig D, Grosse J. Differentiated thyroid cancer: treatment—state of the art. Int J Mol Sci. 2017;18:292.

19. Cipriani NA, Nagar S, Kaplan SP, et al. Follicular thyroid carcinoma: how have histologic diagnoses changed in the last half-century and what are the prognostic implications? Thyroid. 2015;25:1209-1216.

20. Gimm O, Dralle H. Differentiated thyroid carcinoma. In: Surgical Treatment: Evidence-Based and Problem-Oriented. Zuckschwerdt; 2001:1-15.

21. Stenson G, Nilsson IL, Mu N, et al. Minimally invasive follicular thyroid carcinomas: prognostic factors. Endocrine. 2016;53:505-511.

22. Besic N, Schwarzbartl-Peve A, Vidergar-Kralj B, Crnic T, Gazic B, Marolt Music M. Treatment and outcome of 32 patients with distant metastases of Hürthle cell thyroid carcinoma: a single-institution experience. BMC Cancer. 2016;16:162.

23. Ahmadi S, Stang M, Jiang XS, Sosa JA. Hürthle cell carcinoma: current perspectives. Onco Targets Ther. 2016;9:6873-6884.

24. De Melo AS, De Oliveira Rodrigues MF, Marchiori E. Metastatic Hurthle cell cancer. QJM. 2019;112:453-454.

25. Cannon J. The significance of Hurthle cells in thyroid disease. Oncologist. 2011;16: 1380-1387.

26. Perri F, Di Lorenzo G, Della Vittoria Scarpati G, Buonerba C. Anaplastic thyroid carcinoma: a comprehensive review of current and future therapeutic options. World $J$ Clin Oncol. 2011;2:150-157.

27. Romei C, Elisei R. RET/PTC translocations and clinico-pathological features in human papillary thyroid carcinoma. Front Endocrinol (Lausanne). 2012;3:54.

28. Xing M. Clinical utility of RAS mutations in thyroid cancer: a blurred picture now emerging clearer. BMC Med. 2016;14:12.

29. Donati B, Ciarrocchi A. Telomerase and telomeres biology in thyroid cancer. Int $J$ Mol Sci. 2019;20:2887.

30. Zatelli MC, Trasforini G, Leoni S, et al. BRAF V600E mutation analysis increases diagnostic accuracy for papillary thyroid carcinoma in fine-needle aspiration biopsies. Eur J Endocrinol. 2009;161:467-473.

31. Yu XM, Lo CY, Chan WF, Lam KY, Leung P, Luk JM. Increased expression of vascular endothelial growth factor $\mathrm{C}$ in papillary thyroid carcinoma correlates with cervical lymph node metastases. Clin Cancer Res. 2005;11:8063-8069.

32. Wang Y, Ji M, Wang W, et al. Association of the T1799A BRAF mutation with tumor extrathyroidal invasion, higher peripheral platelet counts, and over-expression of platelet-derived growth factor-B in papillary thyroid cancer. Endocr Relat Cancer. 2008;15:183-190.

33. Tuttle RM. Controversial issues in thyroid cancer management. JNucl Med. 2018;59: 1187-1194.

34. Haugen BR, Alexander EK, Bible KC, et al. 2015 American Thyroid Association management guidelines for adult patients with thyroid nodules and differentiated thyroid cancer: The American Thyroid Association Guidelines Task Force on thyroid nodules and differentiated thyroid cancer. Thyroid. 2016;26:1-133.

35. Tuttle RM, Ahuja A, Avram AM, et al. Controversies, consensus, and collaboration in the use of ${ }^{131} \mathrm{I}$ therapy in differentiated thyroid cancer: a joint statement from the American Thyroid Association, the European Association of Nuclear Medicine, the Society of Nuclear Medicine and Molecular Imaging, and the European Thyroid Association. Thyroid. 2019;29:461-470.

36. Lee YM, Jeon MJ, Kim WW, et al. Optimal thyrotropin suppression therapy in lowrisk thyroid cancer patients after lobectomy. J Clin Med. 2019;8:1279.

37. Pirahanchi Y, Toro F, Jialal I. Physiology, thyroid stimulating hormone. National Center for Biotechnology Information website. https:/www.ncbi.nlm.nih.gov/ books/NBK499850/. Updated May 9, 2021. Accessed June 30, 2021.

38. Sodium iodide I 131 solution therapeutic for oral use. U.S. Food and Drug Administration website. https://www.accessdata.fda.gov/drugsatfda_docs/label/2012/ 016515s010lbl.pdf. Updated January 2012. Accessed June 30, 2021.

39. Faugeras L, Pirson AS, Donckier J, et al. Refractory thyroid carcinoma: which systemic treatment to use? Ther Adv Med Oncol. 2018;10:1758834017752853.

40. Nexavar (sorafenib) tablets, oral. U.S. Food and Drug Administration website. https:// www.accessdata.fda.gov/drugsatfda_docs/label/2010/021923s008s0091bl.pdf. Updated October 2010. Accessed June 30, 2021.

41. Lenvima (lenvatinib) capsules, for oral use. U.S. Food and Drug Administration website. http://www.lenvima.com/pdfs/prescribing-information.pdf. Updated December 2020. Accessed June 30, 2021.

42. Retevmo (selpercatinib). Eli Lilly and Company website. https://uspl.lilly.com/ retevmo/retevmo.html\#section-1.3. Updated January 2021. Accessed June 30, 2021.

43. Rothenberg SM, Daniels GH, Wirth LJ. Redifferentiation of iodine-refractory BRAF V600E-mutant metastatic papillary thyroid cancer with dabrafenib-response. Clin Cancer Res. 2015;21:5640-5641.

44. Sun XS, Sun SR, Guevara N, et al. Chemoradiation in anaplastic thyroid carcinomas. Crit Rev Oncol Hematol. 2013;86:290-301. 